\title{
Dos conceptos encontrados de derecho a la cultura en la Constitución de Colombia
}

\author{
Two Conflicting Concepts of the Right to Culture \\ in the Colombian Constitution
}

\author{
Fernando Charria-García ${ }^{1} \bowtie$ \\ 1 Abogado, Universidad Externado de Colombia, Magíster en Estudios Políticos de la Pontificia Universidad \\ Javeriana, Cali, candidato a Doctor en Derecho y Ciencias Sociales de la UNED de España. Docente \\ tiempo completo de la Facultad de Derecho de la Universidad Cooperativa de Colombia, sede Cali, \\ líder del grupo de investigación Humanitas luris. En la actualidad adelanta la investigación "Cultura del \\ derecho de autor en los estudiantes de las universidades y facultades de arte en América Latina".
}

Correo electrónico: fernando.charriag@campusucc.edu.co

Recibido: 2 de marzo del $2018 \quad$ Aprobado: 21 de marzo del $2018 \quad$ Disponible en línea: 1 de abril del 2018

Cómo citar este artículo: Fernando Charria-García. Dos conceptos encontrados de Derecho a la cultura en la Constitución de Colombia. DIXI 27. Abril 2018. doi: https://doi.org/10.16925/2357-5891.2018.01.07

\section{Resumen}

Propósito: el presente trabajo indaga sobre el concepto de cultura de la Declaración Universal de Derechos Humanos de 1948, y el concepto de cultura en la Constitución Política de Colombia de 1991, a la luz de la concepción de bloque de constitucionalidad. Nada de particular podría tener esta referenciación salvo que se trata de dos formas de expresar el concepto de cultura que son diferentes, lo cual supone la generación de una tensión-distensión entre el concepto de cultura expresado en la Constitución Política de Colombia de 1991 y el concepto de cultura en la Declaración de Derechos Humanos de 1948.

Descripción: como es apenas natural, es imprescindible resolver dicha tensión mediante un análisis y un estudio interpretativo cuya importancia radica en que nos debe indicar la manera de resolver la tensión de dos conceptos sobre cultura desde la perspectiva jurídica.

Punto de vista: el presente trabajo ofrece un tipo de interpretación cuya aspiración es dejar una impronta que pueda utilizar el juzgador cuando se deba ocupar de este asunto.

Conclusiones: a la fecha, este concepto no lo ha tomado en cuenta ninguna de las altas cortes judiciales en Colombia, y de manera particular la Corte Constitucional, ente encargado por su naturaleza de la interpretación y el control de las normas constitucionales.

Palabras clave: bloque de constitucionalidad, constitución, cultura, derechos humanos, interpretación. 


\title{
Two Conflicting Concepts of the Right to Culture in the Colombian Constitution
}

\begin{abstract}
Purpose: The present work explores the concept of culture in both the Universal Declaration of Human Rights of 1948 and the Colombian Constitution of 1991, in light of the conception of body of constitutional law. These references could have nothing particular, except that they are two different ways of expressing the concept of culture, which gives rise to a tense/relaxed relationship between the Colombian Constitution of 1991 and the Declaration of Human Rights of 1948.

Description: As it is only natural, it is essential to resolve this tension from a legal perspective through an analysis and an interpretive study of the two concepts of culture.

Point of view: This paper offers a type of interpretation that intends to provide the judge with an impression that can be used when dealing with this matter.

Conclusions: To date, this concept has not been considered by any of the high judicial courts in Colombia, particularly the Constitutional Court, which is in charge of interpreting and controlling constitutional rules.
\end{abstract}

Keywords: body of constitutional law, constitution, culture, human rights, interpretation.

\section{Dois conceitos do direito à cultura confrontados na Constituição da Colômbia}

\section{Resumo}

Objetivo: o presente trabalho explora os conceitos de cultura da Declaração Universal dos Direitos Humanos de 1948 e da Constituição Política da Colômbia de 1991, a partir da concepção do bloco de constitucionalidade. Nada de particular poderia ter essa referência, exceto que são duas maneiras diferentes de expressar o conceito de cultura, o qual gera uma tensão e um relaxamento simultâneos entre 0 conceito de cultura expresso na Constituição Política da Colômbia de 1991 e 0 conceito de cultura da Declaração de Direitos Humanos de 1948.

Descrição: naturalmente, é essencial realizar uma análise e um estudo interpretativo que indiquem, do ponto de vista legal, a maneira de resolver a tensão entre os dois conceitos de cultura.

Ponto de vista: o presente trabalho oferece um tipo de interpretação que aspira a deixar uma marca que o juiz possa usar quando precisar tratar essa questão.

Conclusões: até o momento, este conceito não foi levado em conta por nenhum dos tribunais superiores da Colômbia e, em particular, pelo Tribunal Constitucional, a entidade responsável pela interpretação e 0 controle das normas constitucionais.

Palavras-chave: bloco de constitucionalidade, constituição, cultura, direitos humanos, interpretação. 


\section{Propósito}

El presente escrito plantea las diferentes concepciones de cultura presentes en la Declaración Mundial de Derechos Humanos de 1948, y en nuestra Constitución Política de Colombia de 1991, además de la idea de bloque de constitucionalidad y lo que sobre dicho concepto ha sentenciado la Corte Constitucional, con el objeto de presentar unas conclusiones y una propuesta frente a esta tensión.

La idea de cultura, al estar incorporada en nuestra Constitución, ha dejado de ser un elemento de segunda y ha pasado a ser conformador de la identidad nacional, de manera que ocupa un primerísimo lugar y, por tanto, evidenciar una tensión del término cultura en una y otra referencia jurídica arriba señaladas supone contribuir a su aclaración y consolidación en el derecho colombiano.

\section{DESCRIPCIÓN}

En este aparte se pretende realizar una presentación de los referentes que nos interesan y son fundamento para la construcción conceptual que se ha propuesto, en razón a que se torna muy importante al no encontrarse este concepto involucrado en la anterior Constitución Política de 1886, y sí en cambio en la de 1991 con un sentido central y finalista, como valor y como principio. Para el desarrollo de lo anterior se desarrolla un trabajo de hermenéutica jurídica en busca del sentido de este ámbito temático constitucional.

\section{A. La cultura y los derechos humanos de 1948}

La Declaración Universal de Derechos Humanos, adoptada el 10 de diciembre de 1948, ${ }^{1}$ en el "Preámbulo", en su proclama y en los primeros dos artículos, muestra su fundamentación y sus características, lo cual permite que dicho instrumento adquiera la fuerza de un verdadero tratado.

En la segunda consideración de esta declaración se indica que el desconocimiento y el menosprecio de los derechos humanos han originado actos de

1 Cf. Declaración Universal de Derechos Humanos de 1948 Hchr.org.co. Disponible en: http://www.hchr.org.co/documentoseinformes/documentos/carceles/1_Universales/B\%E1sicos/1_ Generales_DH/1_Declaracion_Universal_DH.pdf barbarie ultrajantes para la conciencia de la humanidad. Con respeto nos apartamos de esa afirmación, pues consideramos que fue el fruto de la reflexión que las barbaries de todo tipo generaron lo que dio paso a estos derechos, ${ }^{2}$ y no lo contrario; es decir, la barbarie no fue fruto del desconocimiento de los derechos humanos.

Ahora bien, en la consideración primera de dicha declaración es evidente el reconocimiento de la dignidad intrínseca del ser humano y la necesidad de unos mínimos básicos como derechos iguales e inalienables para todo el género humano. Por esta razón, es posible comprender mejor la consideración tercera de la declaración, pues destaca la necesidad de que los derechos humanos se positivisen, de modo que tengan efecto, sean medidos, controlados, seguidos, mejorados, etc. A fin de desarrollar esto la consideración séptima proporciona una concepción común de estos derechos y libertades entre los Estados miembros y la ONU, así como en su "proclama", la cual involucra a los individuos y a las instituciones como premisa básica, mientras la consideración quinta nos habla de cómo los pueblos de las Naciones Unidas pueden promover el progreso social y elevar el nivel de vida dentro de un concepto más amplio de la libertad, en procura de relaciones más amistosas entre las naciones.

Lo anterior con el fin de que, mediante la enseñanza y la educación, promuevan el respeto a estos derechos y libertades, y aseguren - por medidas progresivas - su reconocimiento y aplicación universales y efectivos, lo que incluye la estrategia de incorporarlos en un régimen de derecho.

Ahora bien, el supuesto del cual se parte es que estos derechos cobijan a todas las personas sin distinción. Nuestra consideración es que la persona solo es persona en la medida en que se encuentra en una relación con sus semejantes. Se encuentra la vieja concepción aristotélica del zoon politikón, ahora ubicada con unos mínimos de derechos civiles y políticos (pues ellos constituyen la persona), así como de derechos económicos ligados con la libertad, de derechos sociales (que facilitan las libertades de reunión y asociación y aquellas tendientes al mejoramiento de la calidad de vida e involucran asuntos como seguridad social, el trabajo, el tiempo libre y el descanso,

\footnotetext{
2 Cf. Eudoro Echeverri Quintana. Aproximación al bloque de constitucionalidad. Revista Memorando de Derecho 15(X) tercera época. Primer semestre 2006. Págs. 20-21.
} 
entre otros), y de derechos a la educación y la cultura (con los que se constituye, desarrolla y se da garantía de pervivencia al ser humano). Aspectos todos que deben entenderse de manera integral y evitar jerarquías internas, aunque las formas de concretar estos derechos tengan algunas diferencias, de tal manera que los derechos humanos económicos, sociales y culturales tengan como criterio de aplicación la progresividad y que esto entre en relación con la gobernanza. ${ }^{3}$

La cultura se señala en el artículo 27 de la Declaración Universal así: "1. Toda persona tiene derecho a tomar parte libremente en la vida cultural de la comunidad, a gozar de las artes y a participar en el progreso científico y en los beneficios que de él resulten".

En este artículo se habla de "tomar parte", es decir, participar, del latín participare, que significa "dividir" o "partir"; y tomar, del latín capere, que significa "tomar" o" recibir parte de o parte en algo", pues se participa no en la totalidad sino en una parcialidad, en trozos de una totalidad. También se puede entender la locución "tomar parte" como formar parte del todo, lo cual implica integrarse.

Así, entonces, cuando en la Declaración Universal de Derechos Humanos de 1948 se habla de cultura, se entiende que es fundamental para el ser humano y que toma libremente parte de esa cultura.

Ese condicionamiento señalado por la palabra libremente connota un tomar parte que no puede tener coerciones externas que impidan su desarrollo por parte de los Estados frente a las personas, y nos acerca a un principio jurídico superior de la cultura que es la libertad, el cual con respecto a la cultura surge de la valoración que se da a la relación entre cultura y desarrollo de la personalidad. Así, entonces, la cultura en cuanto a su institucionalización y preocupación por parte de los poderes públicos funda la necesidad de una libertad que va más allá de la libertad de expresión, y acoge la libertad de creación cultural cuyo contenido consiste en la creación artística, científica y técnica, que implica un trabajo desde lo estético para lo artístico; un trabajo metódico, racional y sometido a comprobación práctica, para la ciencia; y un trabajo de aplicación para lo útil, tanto de

3 Cf. Gabriel Enrique Arjona Pachón. Derechos culturaLeS en el mundo, Colombia y Bogotá: guía Virtual de las REGULACIONES INTERNACIONALES, NACIONALES Y DISTRITALES EN MATERIA DE DEREChOS Culturales. Secretaría Distrital de Cultura, Recreación y Deporte. (2011). Pág. 30. la ciencia como del arte respecto a la técnica. Es una práctica de resimbolización cuyo contenido presenta ausencia de toda constricción externa, pues cualquier límite desnaturaliza dicha libertad de creación. ${ }^{4}$

\section{B. La cultura y la Constitución Política de Colombia}

La Constitución Política de Colombia de 1991 contó, por primera vez, con un verdadero estatuto de la cultura.

Así, en esta Constitución, el artículo 2 es el primero que incorpora la palabra cultura:

Son fines esenciales del Estado: servir a la comunidad, promover la prosperidad general y garantizar la efectividad de los principios, derechos y deberes consagrados en la Constitución; facilitar la participación de todos en las decisiones que los afectan y en la vida económica, política, administrativa y cultural de la Nación; defender la independencia nacional, mantener la integridad territorial y asegurar la convivencia pacífica y la vigencia de un orden justo. ${ }^{5}$

Este artículo señala los fines del Estado, por ende, los campos económico, político, administrativo y cultural son finalistas.

Luego se encuentran al respecto los artículos 7 y 8 de la Constitución: "Artículo 7. El Estado reconoce y protege la diversidad étnica y cultural de la Nación colombiana. Artículo 8. Es obligación del Estado y de las personas proteger las riquezas culturales y naturales de la Nación". ${ }^{6}$

En el artículo séptimo se afirma la existencia, el reconocimiento y la protección de la diversidad étnica y cultural del país; en el octavo se consagra la protección de las riquezas culturales de la Nación, y se indica algo diferente a lo ya dicho sobre cultura, pues de no ser así estaría usando la palabra cultura - que no utiliza-; por tanto, es probable que se refiera a los valores, las creencias y los estilos de vida culturales, pues se trata de elementos de la concepción de cultura que no se desarrollan en otros acápites

\footnotetext{
4 Cf. Fernando Charria-García. Aproximaciones al Derecho DE la Cultura en Colombia. Unidad Central del Valle del Cauca. (2007). Pág. 32.

5 Véase Constitución Política de Colombia [Const]. Artículo 2. Julio 7 de 1991. (Colombia).

$6 \quad$ Id.
} 
constitucionales, y se fundamente en la definición de cultura de la Declaración de Derechos Culturales de Friburgo. ${ }^{7}$

Por su parte, el artículo 44 de la constitución se refiere a los derechos de los niños:

Son derechos fundamentales de los niños: la vida, la integridad física, la salud y la seguridad social, la alimentación equilibrada, su nombre y nacionalidad, tener una familia y no ser separados de ella, el cuidado y amor, la educación y la cultura, la recreación y la libre expresión de su opinión. Serán protegidos contra toda forma de abandono, violencia física o moral, secuestro, venta, abuso sexual, explotación laboral o económica y trabajos riesgosos. Gozarán también de los demás derechos consagrados en la Constitución, en las leyes y en los tratados internacionales ratificados por Colombia. La familia, la sociedad y el Estado tienen la obligación de asistir y proteger al niño para garantizar su desarrollo armónico e integral y el ejercicio pleno de sus derechos. Cualquier persona puede exigir de la autoridad competente su cumplimiento y la sanción de los infractores. Los derechos de los niños prevalecen sobre los derechos de los demás. ${ }^{8}$

En este artículo la cultura, de forma expresa, se considera un derecho fundamental para los niños.

Luego, el artículo 67 trata de la educación, con referencia a la cultura:

La educación es un derecho de la persona y un servicio público que tiene una función social; con ella se busca el acceso al conocimiento, a la ciencia, a la técnica y a los demás bienes y valores de la cultura. La educación formará al colombiano en el respeto a los derechos humanos, a la paz y a la democracia; y en la práctica del trabajo y la recreación, para el mejoramiento cultural, científico, tecnológico y para la protección del ambiente. El Estado, la sociedad y la familia son responsables de la educación, que será obligatoria entre los cinco y los quince años de edad y que comprenderá como mínimo, un año de preescolar y nueve de educación básica. La educación será gratuita en las instituciones del Estado, sin perjuicio del cobro de derechos académicos a quienes puedan

$7 \quad C f$. Los Derechos Culturales. Declaración de Friburgo. Disponible en http://www.culturalrights.net/descargas/drets_culturals239.pdf

Véase Constitución Política de Colombia [Const], supra, nota 5. Artículo 44 sufragarlos. Corresponde al Estado regular y ejercer la suprema inspección y vigilancia de la educación con el fin de velar por su calidad, por el cumplimiento de sus fines y por la mejor formación moral, intelectual y física de los educandos; garantizar el adecuado cubrimiento del servicio y asegurar a los menores las condiciones necesarias para su acceso y permanencia en el sistema educativo. La Nación y las entidades territoriales participarán en la dirección, financiación y administración de los servicios educativos estatales, en los términos que señalen la Constitución y la ley. ${ }^{9}$

Dos son los aspectos culturales que toca. El primero señala cómo, por medio de la educación, se busca el acceso a los bienes y valores de la cultura. El segundo trata del mejoramiento cultural, el cual se entiende como el progreso de la cultura, principio superior de la cultura que, de acuerdo con Prieto de Pedro, se expresa así: "Se requiere de los poderes públicos del Estado para garantizar la no intervención pública en el ejercicio de la libertad de creación cultural, de la diversidad cultural de la sociedad, y la adopción de medidas eficaces que permitan el acceso de todas las personas a los bienes culturales". ${ }^{10}$

Lo anterior se desarrolla en tres aspectos: 1 . Todos los poderes públicos, en razón al contenido holístico de la cultura y de su carácter general y específico, deben desarrollar la función cultural; 2 . La cultura se concreta como derecho que obliga a una actuación positiva de los poderes públicos; 3 . Los derechos fundamentales como valor superior de toda cultura en correlación con la tolerancia como valor que confiere un carácter universal a las culturas.

La conexión entre educación y cultura permite formular el principio de enculturación democrática mediante el cual la educación tiene por objeto el pleno y libre desarrollo de la personalidad ${ }^{11}$, el fortalecimiento de los derechos y las libertades fundamentales, con un contenido negativo de promover manifestaciones contrarias a los derechos humanos y a los principios democráticos, especialmente de dignidad y tolerancia.

El artículo 68 constitucional señala al respecto: "Los integrantes de los grupos étnicos tendrán derecho a una formación que respete y desarrolle

$9 \quad$ Id. Artículo 67.

10 Véase Jesús Prieto de Pedro. Cultura, culturas y ConsTitución. Congreso de los Diputados y Centro de Estudios Constitucionales. (1994).

11 Son múltiples las sentencias sobre libre desarrollo de la personalidad de nuestra Corte Constitucional: e. g. C-488/02; T-067/98; C-131/14; sU-642/98; C-449/03, etc. 
su identidad cultural". Esto en conexión con el artículo séptimo que, al reconocer la diversidad étnica y cultural, obliga a que se entienda la educación como aquella que se ofrece al respetar y fomentar esta diversidad.

En el artículo 70 se lee:

El Estado tiene el deber de promover y fomentar el acceso a la cultura de todos los colombianos en igualdad de oportunidades, por medio de la educación permanente y la enseñanza científica, técnica, artística y profesional en todas las etapas del proceso de creación de la identidad nacional.

La cultura en sus diversas manifestaciones es fundamento de la nacionalidad. El Estado reconoce la igualdad y dignidad de todas las que conviven en el país. El Estado promoverá la investigación, la ciencia, el desarrollo y la difusión de los valores culturales de la Nación. ${ }^{12}$

El primer inciso nos habla del acceso a la cultu$r a$, concepto basado en el progreso de la cultura que supone la adopción de medidas eficaces para que todas las personas puedan acceder sin distingos a los bienes culturales; lo promueve y lo fomenta la educación.

El segundo inciso es la piedra de "ara" de nuestro estatuto cultural constitucional, la cual considera la cultura como un fundamento de la nacionalidad, base sobre la que descansa la identidad nacional. Esto obliga al Estado a promover "la investigación, la ciencia, el desarrollo y la difusión de los valores culturales de la Nación".

Luego, el artículo 71 señala:

La búsqueda del conocimiento y la expresión artística son libres. Los planes de desarrollo económico y social incluirán el fomento a las ciencias y, en general, a la cultura. El Estado creará incentivos para personas e instituciones que desarrollen y fomenten la ciencia y la tecnología y las demás manifestaciones culturales y ofrecerá estímulos especiales a personas e instituciones que ejerzan estas actividades. ${ }^{13}$

En este punto se ordena incluir en los planes de desarrollo el fomento a la cultura, lo cual implica incentivos para todas las manifestaciones culturales diferentes a la ciencia y la tecnología, así como estímulos a personas e instituciones que ejerzan actividades culturales como resultado de una concepción antropológica de la cultura, lo cual se deduce cuando el artículo indica "las demás manifestaciones culturales", pues significa que existen otras distintas a la ciencia y la tecnología.

Por su parte, el artículo 72 trata de cultura en el sentido en que,

El patrimonio cultural de la Nación está bajo la protección del Estado. El patrimonio arqueológico y otros bienes culturales que conforman la identidad nacional, pertenecen a la Nación y son inalienables, inembargables e imprescriptibles. La ley establecerá los mecanismos para readquirirlos cuando se encuentren en manos de particulares y reglamentará los derechos especiales que pudieran tener los grupos étnicos asentados en territorios de riqueza arqueológica. ${ }^{14}$

El patrimonio cultural es parte de la cultura y ostenta las notas jurídicas de inembargabilidad, inalienabilidad e imprescriptibilidad de todos sus bienes. El artículo 95 afirma:

La calidad de colombiano enaltece a todos los miembros de la comunidad nacional. Todos están en el deber de engrandecerla y dignificarla. El ejercicio de los derechos y libertades reconocidos en esta Constitución implica responsabilidades. Toda persona está obligada a cumplir la Constitución y las leyes. Son deberes de la persona y del ciudadano:

(...)

8. Proteger los recursos culturales y naturales del país y velar por la conservación de un ambiente sano. ${ }^{15}$

Nuestra Constitución señala que esto es una obligación del Estado, las personas y los ciudadanos.

En el artículo 222 se lee: "La ley determinará los sistemas de promoción profesional, cultural y social de los miembros de la Fuerza Pública. En las etapas de su formación, se les impartirá la enseñanza de los fundamentos de la democracia y de los derechos humanos". 16
12 Véase Constitución Política de Colombia [Const], supra, nota 5. Artículo 70.

13 Id. Artículo 71.
Id. Artículo 72.

Id. Artículo 95.

Id. Artículo 222 
Señalamiento para que la ley determine un sistema de promoción cultural de los miembros de la Fuerza Pública, mandato que obliga a que el Congreso expida dicha ley.

Luego, en el artículo 305 declara: "Son atribuciones del gobernador: (...) 6. Fomentar de acuerdo con los planes y programas generales, las empresas, industrias y actividades convenientes al desarrollo cultural, social y económico del departamento que no correspondan a la Nación y a los municipios".

Entre las atribuciones de los mandatarios departamentales, se encuentra el fomento de empresas, industrias y actividades propicias al desarrollo cultural, acorde con los planes de desarrollo.

Continúa con el artículo 310:

El Departamento Archipiélago de San Andrés, Providencia y Santa Catalina (...) Mediante ley aprobada por la mayoría de los miembros de cada cámara se podrá limitar el ejercicio de los derechos de circulación y residencia, establecer controles a la densidad de la población, regular el uso del suelo y someter a condiciones especiales la enajenación de bienes inmuebles con el fin de proteger la identidad cultural de las comunidades nativas y preservar el ambiente y los recursos naturales del Archipiélago. ${ }^{17}$

Sobre este archipiélago, elevado a departamento por tener un referente étnico cultural específico, la Constitución impone que una ley limite la circulación, la residencia, la densidad de población, los usos del suelo y la enajenación de bienes, a fin de proteger la identidad cultural de sus comunidades nativas.

Luego, el artículo 311 refiere:

Al municipio como entidad fundamental de la división político-administrativa del Estado le corresponde prestar los servicios públicos que determine la ley, construir las obras que demande el progreso local, ordenar el desarrollo de su territorio, promover la participación comunitaria, el mejoramiento social y cultural de sus habitantes y cumplir las demás funciones que le asignen la Constitución y las leyes. ${ }^{18}$

Este mandato obliga al municipio a la promoción y el mejoramiento cultural al consagrar una concepción progresiva de ese mandato.

17 Id. Artículo 310.

18 Id. Artículo 311.
Más adelante, en el artículo 313 se lee que "corresponde a los concejos: (...) 9. Dictar las normas necesarias para el control, la preservación y defensa del patrimonio ecológico y cultural del municipio.

Los concejos municipales por mandato constitucional deben "dictar las normas necesarias" para el control, la preservación y la defensa del patrimonio cultural del municipio, ya que es el municipio el que conoce su patrimonio cultural y sus necesidades.

Luego se encuentra el artículo 333:

La actividad económica y la iniciativa privada son libres, dentro de los límites del bien común. Para su ejercicio, nadie podrá exigir permisos previos ni requisitos, sin autorización de la ley.

(...)

La ley delimitará el alcance de la libertad económica cuando así lo exijan el interés social, el ambiente y el patrimonio cultural de la Nación. ${ }^{19}$

Señala así, de forma expresa - como límite a la libertad económica- el del patrimonio cultural.

Nuestra Constitución contiene un grupo de 15 artículos que tratan de la cultura, lo que es suficiente para hablar de un verdadero estatuto de cultura, entendiendo que dicho concepto se configura en un sentido holístico más que en uno polisémico.

\section{Bloque de constitucionalidad}

La idea de bloque de constitucionalidad es relativamente novedosa en Colombia, pues surge después de la Constitución de 1991; algunos autores indican que esto se da desde 1995.

El profesor Rodrigo Uprimny afirma: "El bloque de constitucionalidad hace referencia a normas constitucionales que no se encuentran en la Constitución Política". ${ }^{20}$ Son normas que no necesariamente se encuentran en el texto constitucional, y pueden estar en otros documentos de carácter internacional. La doctrina lo entiende como "el conjunto de normas que configuran una unidad constitucional que es empleada como parámetro de constitucionalidad de

\footnotetext{
19 Id. Artículo 312.

20 Véase Rodrigo Uprimny. El BLOQUe DE CONSTITUCIONALIDAD EN COlOMbia. CURSO DE FORMACIÓN DE PROMOTORES/AS DE DERECHOS HUMANOS LIBERTAD SINDICAL Y TRABAJO DECENTE. Ed: Universidad-Ens Colombia. (2005). Pág. 2.
} 
las restantes normas del ordenamiento". ${ }^{21}$ Esto indica que las constituciones no son códigos cerrados, pues pueden hacer remisiones, expresas o tácitas, a otras reglas y principios que, sin estar en la Constitución, tienen importancia en la práctica constitucional, porque la propia constitución así lo determina, incluso, si son referentes expresamente señalados o indeterminados. Así, entonces, es por la mera voluntad y la determinación de la Constitución que se da nacimiento al bloque de constitucionalidad.

Lo anterior genera obligaciones en todos los operadores jurídicos, y obliga a tener en cuenta normas que están más allá de la Constitución.

\section{La Corte Constitucional señala:}

Las normas del bloque operan como disposiciones básicas que reflejan los valores y principios fundacionales del Estado y también regulan la producción de las demás normas del ordenamiento doméstico. Dado el rango constitucional que les confiere la carta, las disposiciones que integran el bloque superior cumplen la cuádruple finalidad que les asigna Bobbio, a saber, servir de i) regla de interpretación respecto de la dudas que puedan suscitarse al momento de su aplicación; ii) la de integrar la normatividad cuando no exista norma directamente aplicable al caso; iii) la de orientar las funciones del operador jurídico; y iv) la de limitar la validez de las regulaciones subordinadas. ${ }^{22}$

Por su parte, el profesor Uprimny afirma:

La idea misma del bloque de constitucionalidad y los debates que le están asociados son pues tan antiguos como la propia justicia constitucional. Sin embargo, la categoría con su nombre e intentos de precisión dogmática, es más reciente y se origina en la práctica del Consejo Constitucional francés en el marco de la llamada v República. (...) El punto de base de esa verdadera "revolución constitucional", como la denominan algunos autores, fue que ese tribunal atribuyó pleno valor constitucional al preámbulo, a pesar de que los constituyentes de 1958 no quisieron que éste tuviera ninguna fuerza jurídica. Esa opción hermenéutica fue de enorme trascendencia pues, a pesar de su brevedad, ese preámbulo hace remisiones normativas que, de ser tomadas en serio, aumentan considerablemente la extensión y la densidad normativa de la Constitución. En efecto, ese texto señala que "el pueblo francés proclama solemnemente su adhesión a los derechos humanos y a los principios de la soberanía nacional tal y como fueron definidos por la Declaración de 1789, confirmada y completada por el Preámbulo de la Constitución de $1946{ }^{23}$

Se ha manifestado que "se trata de una herramienta de integración del derecho internacional en el orden constitucional colombiano y por ende, de un medio de ampliación de la normatividad constitucional, para el control de la normatividad jerárquicamente inferior e, igualmente, para la garantía de los derechos humanos en el país". ${ }^{24}$

El bloque de constitucionalidad, entonces, se introdujo expresamente en nuestra Constitución Política de 1991:

Los tratados y convenios internacionales ratificados por el Congreso, que reconocen los derechos humanos y que prohíben su limitación en los estados de excepción, prevalecen en el orden interno.

Los derechos y deberes consagrados en esta Carta, se interpretarán de conformidad con los tratados internacionales sobre derechos humanos ratificados por Colombia.

El Estado colombiano puede reconocer la jurisdicción de la Corte Penal Internacional en los términos previstos en el Estatuto de Roma adoptado el 17 de julio de 1998 por la Conferencia de Plenipotenciarios de las Naciones Unidas y, consecuentemente, ratificar este tratado de conformidad con el procedimiento establecido en esta Constitución. La admisión de un tratamiento diferente en materias sustanciales por parte del Estatuto de Roma con respecto a las garantías contenidas en la Constitución tendrá efectos exclusivamente dentro del ámbito de la materia regulada en él. ${ }^{25}$

Esta incorporación en una Constitución abierta tiene ventajas y desventajas, así como genera sin lugar a dudas su mayor versatilidad.
21 Véase Luis Andrés Fajardo Arturo. Contenido y alcance jurisprudencial del bloque de constitucionalidad en Colombia. Revista Civilizar Ciencias Sociales y Humanas 7(13). 2007. Pág. 79.

22 Véase Corte Constitucional de Colombia. Sentencia C-067 de 2003. (MP Marco Monroy Cabra: febrero 4 de 2003).
23 Véase Rodrigo Uprimny, supra, nota 20.

24 Véase Luis Andrés Fajardo Arturo, supra, nota 21. Pág. 16.

25 Véase Constitución Política de Colombia [Const], supra, nota 5. Artículo 93. 
También juega un papel importante el artículo 53 de la Constitución: "El Congreso expedirá el estatuto del trabajo. La ley correspondiente tendrá en cuenta por lo menos los siguientes principios mínimos fundamentales: (...) Los convenios internacionales del trabajo debidamente ratificados, hacen parte de la legislación interna". ${ }^{26}$

Otro tanto importa al respecto el artículo 94: “La enunciación de los derechos y garantías contenidos en la Constitución y en los convenios internacionales vigentes, no debe entenderse como negación de otros que, siendo inherentes a la persona humana, no figuren expresamente en ellos". ${ }^{27}$

Este artículo consagra la cláusula de los derechos innominados. Cierra este grupo constitucional el artículo 214:

Los Estados de Excepción a que se refieren los artículos anteriores se someterán a las siguientes disposiciones:

(...)

2. No podrán suspenderse los derechos humanos ni las libertades fundamentales. En todo caso se respetarán las reglas del derecho internacional humanitario. Una ley estatutaria regulará las facultades del Gobierno durante los estados de excepción y establecerá los controles judiciales y las garantías para proteger los derechos, de conformidad con los tratados internacionales. Las medidas que se adopten deberán ser proporcionales a la gravedad de los hechos. ${ }^{28}$

Se considera que ni siquiera en momentos de gran dificultad y crisis como los estados de excepción, se pueden desconocer ni suspender los derechos humanos ni el derecho internacional humanitario.

La incorporación del bloque de constitucionalidad sirvió para permitir un acercamiento y uso que, de acuerdo con Uprimny, ${ }^{29}$ supuso cuatro sentencias que estructuraron su desarrollo:

- La Sentencia T-002 de 1992 analiza los criterios que permiten determinar si un derecho es o no fundamental $y$, por ende, si es o no tutelable por encontrarse en un tratado de derechos humanos.

- La Sentencia T-409 de 1992 sobre los Convenios de Ginebra de derecho humanitario, por la cual se puso límites a la obediencia militar.

26 Id. Artículo 53.

27 Id. Artículo 94.

28 Id. Artículo 214.

29 Véase Rodrigo Uprimny, supra, nota 20.
- La Sentencia C-574 de 1992 revisó la constitucionalidad del "Protocolo I a los Convenios de Ginebra,", ${ }^{30}$ y señaló con base en los artículos 93, 94 y 214 de la Constitución, que la Carta había conferido a esa normatividad humanitaria un rango supraconstitucional, de modo que operó una incorporación automática de dicha normatividad al ordenamiento interno.

- La Sentencia T-426 de 1992, la cual tuvo en cuenta la Declaración Universal de Derechos Humanos y el Pacto de Derechos Económicos, Sociales y Culturales para reconocer como fundamental un derecho que no aparecía de manera expresa en la Carta: el llamado derecho a la subsistencia o derecho al mínimo vital.

\section{El bloque de constitucionalidad en sentido estricto y lato ${ }^{31}$}

Uno de los desarrollos importantes que ha permitido el bloque de constitucionalidad es una integración con ciertas características, al precisarse que "siempre que se habla de bloque de constitucionalidad, se hace porque una norma Constitucional así lo ordena y exige su integración, de suerte que la violación de cualquier norma que lo conforma se resuelve en últimas en una violación del Estatuto Superior" ${ }^{32}$ Sin embargo, es en la Sentencia C-358 de 1997, ${ }^{33}$ fundamentos 3 y 4, en la que la Corte distingue entre las "normas situadas a nivel constitucional" como los convenios de derecho internacional humanitario que tienen jerarquía y fuerza constitucional, y otras disposiciones que no tienen rango constitucional, pero que la misma Constitución ordena que las respeten las leyes ordinarias, "tal y como sucede con las leyes orgánicas y estatutarias en determinados campos". En la Sentencia C-191 de 1998 la Corte puntualiza los criterios que permiten entender un bloque de constitucionalidad en sentido estricto, pues debe tratarse de normas de rango constitucional, mientras que en sentido lato son "parámetros" de constitucionalidad. Así, en el fundamento 5, se lee:

\footnotetext{
30 Cf. Declaración y Programa de Acción de Viena. OHChr. org. Disponible en: http://www.ohchr.org/Documents/Events/ OHCHR20/VDPA_booklet_Spanish.pdf

31 Cf. Eudoro Echeverri Quintana, supra, nota 2.

32 Véase Corte Constitucional de Colombia. Sentencia C-578 de 1995. (MP Jaime Córdoba Triviño: diciembre 4 de 1995).

33 Véase Corte Constitucional de Colombia. Sentencia C-358 de 1997. (MP Eduardo Cifuentes Muñoz: agosto 5 de 1995).
} 
Resulta posible distinguir dos sentidos del concepto de bloque de constitucionalidad. En un primer sentido de la noción, que podría denominarse bloque de constitucionalidad stricto sensu, se ha considerado que se encuentra conformado por aquellos principios y normas de valor constitucional, los que se reducen al texto de la Constitución propiamente dicha y a los tratados internacionales que consagren derechos humanos cuya limitación se encuentre prohibida durante los estados de excepción (C. N., artículo 93). ${ }^{34}$

Para el sentido lato, la misma sentencia señaló:

Más recientemente, la Corte ha adoptado una noción lato sensu del bloque de constitucionalidad, según la cual aquel estaría compuesto por todas aquellas normas, de diversa jerarquía, que sirven como parámetro para llevar a cabo el control de constitucionalidad de la legislación. Conforme a esta acepción, el bloque de constitucionalidad estaría conformado no sólo por el articulado de la Constitución sino, entre otros, por los tratados internacionales de que trata el artículo 93 de la Carta, por las leyes orgánicas y, en algunas ocasiones, por las leyes estatutarias.

Así, entonces, las normas del bloque de constitucionalidad son las que se integran porque la Constitución expresamente lo señala, pues los tratados en general no forman parte de este bloque de constitucionalidad, ya que la Constitución no señala en sus disposiciones que el conjunto de los tratados ratificados por Colombia debe ser tenido en cuenta por la Corte al examinar la constitucionalidad de las leyes. Asimismo, porque el hecho de que la norma Pacta Sunt Servanda guíe las relaciones del país y obligue a su acatamiento, no significa que las normas legales contrarias a los tratados deban ser consideradas inconstitucionales, pues se convierte en un imposible confrontar la validez de las normas legales frente a la totalidad de los tratados. Significaría esto que la Corte Constitucional revisara las normas impugnadas en referencia a los tratados ratificados por Colombia, lo que es desmesurado e inviable. También con base en el principio de reciprocidad muchos convenios exigen esta condición para que sus cláusulas se apliquen, por lo cual su aplicabilidad a un caso concreto dependerá del cumplimiento de esa exigencia, de manera que mal podría excluirse

34 Véase Corte Constitucional de Colombia. Sentencia C-191 de 1998. (мP Marco Monroy Cabra: mayo 6 de 2003). de forma permanente del ordenamiento una ley por violar un tratado cuya aplicabilidad está sujeta a contingencias. No es factible por la vía del control constitucional abstracto y, en términos generales, declarar la inexequibilidad de una norma que contradiga un tratado. ${ }^{35}$ Estas determinaciones se ampliaron luego al incluir los tratados de límites, los convenios de la Organización Internacional del Trabajo (OIT), así como las decisiones y la jurisprudencia de las instancias internacionales de derechos humanos.

Los tratados limítrofes tienen una jerarquía especial que les otorga el artículo 102 de la Constitución, en el cual se señala que el territorio colombiano se encuentra delimitado por dichos convenios. ${ }^{36}$ Lo reitera la Corte constitucional cuando señala que las leyes aprobatorias de los tratados pueden ser demandadas, pero no las que hacen referencia a los límites, dada la jerarquía que les otorgó el artículo 102 de la Carta. ${ }^{37}$ Por último, en el estudio de constitucionalidad del convenio con Honduras sobre la frontera marítima, los tratados perfeccionados sobre fronteras no pueden ser demandados por ser bloque de constitucionalidad. ${ }^{38}$

La Corte Constitucional también señaló que los convenios de la ort hacen parte del bloque de constitucionalidad, ${ }^{39}$ en razón a que los tratados que reconocen derechos laborales en desarrollo del artículo 93 constitucional no pueden suspenderse durante estados de excepción, de modo que se insiste en que la interpretación y la aplicación de los derechos laborales implican los tratados internacionales ratificados como integrados a la Constitución.

En relación con los derechos humanos, la Corte Constitucional señala cómo a pesar de que algunos de ellos prevean su suspensión en estados de excepción (tal el caso del artículo 12 del Pacto Internacional de los Derechos Civiles y Políticos, y el 22 de la Convención Interamericana), con base en el inciso

35 Véase Corte Constitucional de Colombia. Sentencia C-358 de 1997, supra, nota 34

36 Cf. Corte Constitucional de Colombia. Sentencia C-191 de 1998, supra, nota 35.

37 Cf. Corte Constitucional de Colombia. Sentencia c-400 de 1998. (MP Alejandro Martínez Caballero: agosto 10 de 1998).

38 Cf. Corte Constitucional de Colombia. Sentencia C-1022 de 1999. (MP Alejandro Martínez Caballero: diciembre 16 de 1999).

39 Cf. Corte Constitucional de Colombia. Sentencia T-568 de 1999. (MP Carlos Gaviria Díaz: agosto 10 de 1999); y Sentencia C-567 de 2000. (MP Alfredo Beltrán Sierra: mayo 17 de 2000). 
segundo del artículo 93 de la Carta, ${ }^{40}$ para efectos de interpretación, estas normas tienen carácter prevalente en el orden interno, de manera que forman parte del bloque de constitucionalidad. ${ }^{41}$

Respecto a la jurisprudencia en las competencias judiciales internacionales de derechos humanos, en la Sentencia C- 0100 de 2000 se señala que la doctrina elaborada por la Corte Interamericana de Derechos Humanos, como organismo especializado y autorizado para la interpretación de estos derechos, constituye criterio hermenéutico relevante para establecer el sentido de las normas constitucionales sobre derechos fundamentales. ${ }^{42}$

Existen, según el profesor Uprimny, ${ }^{43}$ cinco técnicas básicas de remisión para determinar el bloque de constitucionalidad: 1. La remisión a textos cerrados y definidos, como cuando varias constituciones latinoamericanas incorporan la Convención Interamericana; 2 . El reenvío a textos cerrados, cuya determinación suscita polémicas e incertidumbres, como cuando el "Preámbulo" de la Constitución de Francia de 1946 (que hace parte del bloque por la remisión del "Preámbulo" de la Carta de 1958) a su vez remite "a los principios fundamentales reconocidos por las leyes de la República", pues no se sabe taxativamente cuáles son esas leyes ni esos principios; 3. La remisión a textos por desarrollar, como cuando la Constitución española se refiere a los estatutos de autonomía que serían ulteriormente aprobados; 4 . Las remisiones abiertas a valores y principios, como, por ejemplo, la cláusula de derechos innominados de la Ix enmienda de la Constitución estadounidense; y 5. La remisión a otros valores por medio del uso de conceptos particularmente indeterminados, como, por ejemplo, la cláusula del debido proceso sustantivo en Estados Unidos, en el que los debates en torno a la integración del bloque se confunden con los problemas de interpretación.

En suma, se puede precisar que hacen parte del bloque en sentido estricto:

40 "Los derechos y deberes consagrados en esta Carta, se interpretarán de conformidad con los tratados internacionales sobre derechos humanos ratificados por Colombia”.

41 Cf. Corte Constitucional de Colombia. Sentencia t-483 de 1999. (MP Antonio Barrera Carbonell: julio 8 de 1999).

42 Las sentencias T-568 de 1999 y C-010 de 2000 de la Corte Constitucional son vitales por la utilización del bloque de constitucionalidad de manera extensa. Esto incluye también la SENTENCIA C- 406 de 1996.

43 Véase Rodrigo Uprimny, supra, nota 20.
- El preámbulo.

- El articulado constitucional.

- Los tratados de límites ratificados por Colombia.

- Los tratados de derecho humanitario.

- Los tratados ratificados por Colombia que reconocen derechos intangibles.

- Los artículos de los tratados de derechos humanos ratificados por Colombia, cuando se trate de derechos reconocidos por la Carta.

- La doctrina elaborada por los tribunales internacionales en relación con esas normas internacionales. Se incluyen, específicamente, los convenios de la oit y la doctrina elaborada por los órganos de control de esa organización internacional.

Para el bloque constitucional en sentido lato, además:

- Leyes estatutarias.

- Leyes orgánicas, en lo pertinente.

\section{La contradictio in terminis del concepto cultura entre la Declaración de Derechos Humanos de 1948 y la Constitución Política de Colombia de 1991}

La Declaración Universal de Derechos Humanos de 1948 señala en su artículo 27: "1. Toda persona tiene derecho a tomar parte libremente en la vida cultural de la comunidad, a gozar de las artes y a participar en el progreso científico y en los beneficios que de él resulten". ${ }^{4}$

Por su parte, la Constitución Política de 1991 declara en el artículo 2:

Son fines esenciales del Estado: servir a la comunidad, promover la prosperidad general y garantizar la efectividad de los principios, derechos y deberes consagrados en la Constitución; facilitar la participación de todos en las decisiones que los afectan y en la vida económica, política, administrativa y cultural de la Nación; defender la independencia nacional, mantener la integridad territorial y asegurar la convivencia pacífica y la vigencia de un orden justo.

Las autoridades de la República están instituidas para proteger a todas las personas residentes en Colombia, en su vida, honra, bienes, creencias, y demás derechos

44 Véase Declaración Universal de Derechos Humanos de 1948, supra, nota 1. 
y libertades, y para asegurar el cumplimiento de los deberes sociales del Estado y de los particulares. ${ }^{45}$

Tenemos así dos manifestaciones que positivizan la cultura en un texto, pero estructuradas de manera diferente. En la Declaración Universal de Derechos Humanos se dice que las personas tienen derecho a "tomar parte libremente en la vida cultural de la comunidad". Tomar parte significa participar en el diseño de las políticas, participar en las decisiones, en las manifestaciones culturales como creador, en las manifestaciones como receptor pasivo de las expresiones creativas y patrimoniales, como crítico y experto de los diferentes elementos que componen la cultura, como gestor de esas diferentes manifestaciones, etc. Esto nos obliga saber a qué nos referimos con el término cultura.

El rastreo sobre los conceptos de cultura puede ser desmesurado, e incluir desde la que estructuró Taylor: "Cultura (...) es ese todo complejo que incluye el conocimiento, las creencias, el arte, la moral, el derecho, la costumbre y cualesquiera otros hábitos y capacidades adquiridos por el hombre como miembro de la sociedad"; 46 hasta la de Boas de 1930: "La cultura incluye todas las manifestaciones de los hábitos sociales de una comunidad, las reacciones del individuo en la medida en que se ven afectadas por las costumbres del grupo en que vive, y los productos de las actividades humanas en la medida en que se ven determinadas por dichas costumbres" ${ }^{\prime 47}$; o la de la Ley 397 de 1997: "Cultura es el conjunto de rasgos distintivos, espirituales, materiales, intelectuales y emocionales que caracterizan a los grupos humanos y que comprende, más allá de las artes y las letras, modos de vida, derechos humanos, sistemas de valores, tradiciones y creencias" ${ }^{\prime 8}$ (esta última es la misma de la Unesco). O bien la de Friburgo: "El término 'cultura' abarca los valores, las creencias, las convicciones, los idiomas, los saberes y las artes, las tradiciones, instituciones y modos de vida por medio de los

\footnotetext{
45 Véase Constitución Política de Colombia [Const], supra, nota 5. Artículo 2.

46 Véase J. S. Kahn. El concepto de cultura: textos fundamentales. Editorial Anagrama. (1975).

47 Id.

48 Véase Ley 397 de 1997. Por la cual se desarrollan los artículos 70, 71 y 72 y demás artículos concordantes de la Constitución Política y se dictan normas sobre patrimonio cultural, fomentos y estímulos a la cultura, se crea el Ministerio de la Cultura y se trasladan algunas dependencias. Agosto 7 de 1997. Do 43102. Artículo 1, numeral 1
}

cuales una persona o un grupo expresa su humanidad y los significados que da a su existencia y a su desarrollo". ${ }^{49}$

La definición de Friburgo capta nuestro interés, ya que es posterior y está estructurada para los derechos culturales, por lo que la acogeremos. Entonces podríamos señalar que cuando hablamos de participar en la vida cultural, estaríamos significando un participar de valores, creencias, convicciones, saberes, artes, tradiciones, instituciones y modos de vida por los cuales una persona expresa su humanidad, así como de los significados que da a su existencia y a su desarrollo.

Así, tenemos la participación en ocho grandes aspectos, uno de los cuales serían los modos de vida cuya determinación sería muy compleja. Por tanto, tomar parte es participar en las diferentes formas que señalábamos y que supondrían múltiples grados y diversos requerimientos.

Sin embargo, en nuestra Constitución, en el artículo segundo, la idea de cultura tiene la siguiente enunciación: "Facilitar la participación de todos en las decisiones que los afectan y en la vida económica, política, administrativa y cultural de la Nación". ${ }^{50}$

Esta enunciación es diferente a la de la Declaración Universal de Derechos Humanos, lo cual suscita la siguiente pregunta: ¿Si se tiene en cuenta el bloque de constitucionalidad en sentido estricto, la Declaración Universal de Derechos Humanos forma parte integrante de nuestra Constitución y es norma constitucional?

Tendríamos así dos referencias a la cultura en el mismo texto, y nótese que no hablamos de los desarrollos de dichos conceptos.

En el primer caso tenemos: "Tomar parte libremente en la vida cultural de la comunidad". En el segundo: "Facilitar la participación de todos en las decisiones que los afectan y en la vida (...) cultural".

$\mathrm{Si}$, entonces, tenemos dos textos, a cuál de ellos referirnos en relación con la acepción de cultura que ha cobrado mayor importancia en la actual Constitución?

Frente a lo anterior es necesario un trabajo de interpretación de estos textos, un acercamiento a fin de descomponerlo en sus segmentos de sentido. Así, el primer segmento de sentido dice: "Facilitar la participación de todos en las decisiones que los afectan", el cual podría completarse al señalar que tales

\footnotetext{
49 Véase Declaración de Friburgo, supra, nota 7.

50 Véase Constitución Política de Colombia [Const], supra, nota 5. Artículo 2.
} 
decisiones estarían en relación con la vida cultural de la Nación, de modo que presenta la siguiente opción: "Facilitar la participación de todos en las decisiones que los afectan (...) en la vida cultural de la Nación".

Este tipo de participación sería a todas luces un verdadero reduccionismo con respecto a lo señalado en la Declaración Universal de Derechos Humanos de 1948, pues señala: "Derecho a tomar parte libremente en la vida cultural de la comunidad".

Participar en las decisiones de la vida cultural es totalmente reducido con respecto a participar en la vida cultural, pues las decisiones son solo un aspecto de la vida cultural. De esto resultaría que nuestro texto constitucional se vería necesariamente ampliado y complementado con el de la Declaración Universal de Derechos Humanos.

No obstante, si consideramos: "Facilitar la participación de todos en las decisiones que los afectan y en la vida económica, política, administrativa y cultural de la Nación", podríamos decir que estamos ante dos segmentos de sentido. Uno, formado por: "Facilitar la participación de todos en las decisiones que los afectan"; y otro formado por: "Facilitar la participación de todos en (...) la vida (...) cultural de la Nación”. ¿ Por qué separar este segmento de sentido? Porque lo que se predica es facilitar la participación de todos.

En segundo lugar, esta acción se reúne con la otra que está diferenciada por la partícula "y", como elemento de unión, pues se utiliza con el propósito de unir cláusulas en concepto afirmativo.

Con esto se predican diversos aspectos de la vida, lo que incluye el de la vida cultural. Así, la diferencia entre el sentido de nuestro texto constitucional y el de la Declaración Universal de Derechos Humanos de 1948 se reduce.

En nuestro texto constitucional se pretende "facilitar la participación", de manera que el que actúa como facilitador es el Estado, por estar este artículo en relación con sus fines, y es entonces un fin del Estado facilitar la participación de todos en la vida cultural de la Nación.

En este caso, la diferencia entre ambos textos es sutil, pues en el texto de la Declaración Universal de Derechos Humanos se trata de un "derecho a tomar parte libremente en la vida cultural de la comunidad", mientras que en el nuestro es una facilitación de la participación de la vida cultural de la Nación. En ambos casos se trata de la vida cultural, y en ambos casos es un referente social, acaso más amplio el de nuestra Constitución; sin embargo, lo que sí importa es que en el texto de la Declaración Universal de
Derechos Humanos se toma parte libremente, es decir, la condición de la participación es la libertad de cada persona, elemento que no se encuentra en nuestro texto constitucional y obliga a la siguiente consideración:

La libertad en relación con la cultura surge de la valoración que se da a la relación entre cultura y desarrollo de la personalidad. La cultura en cuanto a su institucionalización y preocupación por parte de los poderes públicos afirma la necesidad de una libertad radical que va más allá de la libertad de expresión. La libertad de creación cultural cuyo objeto y contenido consiste en la creación artística, científica y técnica, lo cual implica un trabajo desde lo estético para lo artístico; un trabajo metódico, racional y sometido a comprobación práctica, para la ciencia; y un trabajo de aplicación para lo útil, tanto de la ciencia como del arte, respecto de la técnica, entonces, esta libertad desde su aspecto dinámico implica una práctica de resimbolización cuyo contenido es de ausencia de toda constricción externa sobre dicha actividad de creación y su proceso de creatividad, en desarrollo de lo cual, cualquier límite puede desnaturalizar y negar la libertad de creación lo que no se niega en relación con la comunicación de esa creación, donde podrán aparecer límites relativos por razón de la protección de la infancia, la juventud, por razón de evitar la pornografía, etc., cuya titularidad, sin limitación alguna, está en las personas físicas. ${ }^{51}$

Por último, tendríamos esta otra opción de sentido: "Facilitar la participación de todos en las decisiones que los afectan y en la vida económica, política, administrativa y cultural de la Nación".

La presentación de esta tercera manera se da porque las dos unidades de sentido que hemos analizado están precisamente relacionadas por la letra " $y$ ", conjunción copulativa que sirve para unir palabras o cláusulas en concepto afirmativo. Esto nos llevaría al siguiente entendimiento: en nuestra Constitución la facilitación de la participación de todos se lleva a cabo con respecto a las decisiones que afectan la vida cultural de la Nación y de la misma vida cultural de la Nación. Se trata de decisión y vida cultural.

En la Declaración Universal de Derechos Humanos se trata de tomar parte de la vida cultural, acción cualificada con la libertad, por tanto, nuestro texto constitucional debe complementarse

51 Véase Fernando Charria-García, supra, nota 4. 
precisamente con este tipo de condicionamiento que es la libertad.

\section{Punto DE VISTA}

Dadas las anteriores descripciones, señalamos que, si se tiene en cuenta que sobre la constitucionalización de la palabra cultura no existía precedente sino hasta la actual Constitución de 1991, es necesario realizar un análisis de un concepto que procura un señalamiento de carácter finalista del Estado, lo que es una necesidad inaplazable y cuya reflexión debe contribuir a la búsqueda de sentido en conformidad con el bloque de constitucionalidad. No hacerlo sería errar, pues se dejaría como vigente una concepción reduccionista de cultura en nuestra Carta Política Fundamental y, por ende, de la finalidad del Estado.

\section{Conclusiones}

Podemos concluir que, al incorporar el concepto de cultura en nuestro texto constitucional de 1991, se incluyó en el artículo segundo que señala los fines del Estado; sin embargo, al desarrollar el concepto de bloque de constitucionalidad en sentido estricto, la Declaración Universal de Derechos Humanos es parte de nuestra Constitución.

No encontramos una contradicción de dos términos y consecuentes conceptos, sino, de la necesaria complementación de estos que se fusionan en uno solo en razón al bloque de constitucionalidad, de tal manera que, cuando nuestro texto constitucional señala como fines del Estado respecto de la cultura facilitar la participación, deberá entenderse como una facilitación de la participación libre en las decisiones que afecten la vida cultural y la misma vida cultural de la Nación.

\section{REFERENCIAS}

Constitución Política de Colombia [Const]. Artículo 2. Julio 7 de 1991. (Colombia).

Corte Constitucional de Colombia. Sentencia C-358 de 1997. (mP Eduardo Cifuentes Muñoz: agosto 5 de 1995).
Corte Constitucional de Colombia. Sentencia C-578 de 1995. (мP Jaime Córdoba Triviño: diciembre 4 de 1995).

Corte Constitucional de Colombia. Sentencia C-400 de 1998. (MP Alejandro Martínez Caballero: agosto 10 de 1998).

Corte Constitucional de Colombia. Sentencia t-483 de 1999. (MP Antonio Barrera Carbonell: julio 8 de 1999).

Corte Constitucional de Colombia. Sentencia t-568 de 1999. (MP Carlos Gaviria Díaz: agosto 10 de 1999).

Corte Constitucional de Colombia. Sentencia C-1022 de 1999. (MP Alejandro Martínez Caballero: diciembre 16 de 1999).

Corte Constitucional de Colombia. Sentencia C-567 de 2000. (MP Alfredo Beltrán Sierra: mayo 17 de 2000).

Corte Constitucional de Colombia. Sentencia C-067 de 2003. (MP Marco Monroy Cabra: febrero 4 de 2003).

Declaración Universal de Derechos Humanos de 1948. Hchr.org.Co. Disponible en: http://www.hchr.org. co/documentoseinformes/documentos/carceles/1_ Universales/B\%E1sicos/1_Generales_DH/1_Declaracion_Universal_DH.pdf

Declaración y Programa de Acción de Viena. OHChr.org. Disponible en: http://www.ohchr.org/Documents/ Events/OHCHR20/VDPA_booklet_Spanish.pdf

Eudoro Echeverri Quintana. Aproximación al bloque de constitucionalidad. Revista Memorando de DereСно 15(X) tercera época, primer semestre, 2006. Págs. 20-21.

Fernando Charria-García. Aproximaciones al Derecho DE la cultura en Colombia. Unidad Central del Valle del Cauca. (2007). Pág. 32.

Gabriel Enrique Arjona Pachón. Derechos culturales en el mundo, Colombia y Bogotá: guía Virtual dE LAS REGULACIONES INTERNACIONALES, NACIONALES Y DISTRITALES EN MATERIA DE DERECHOS CULTURALES. Secretaría Distrital de Cultura, Recreación y Deporte. (2011).

Jesús Prieto de Pedro. Cultura, culturas y ConstituCIón. Congreso de los Diputados y Centro de Estudios Constitucionales. (1994).

Ley 397 de 1997. Por la cual se desarrollan los artículos 70, 71 y 72 y demás artículos concordantes de la Constitución Política y se dictan normas sobre patrimonio cultural, fomentos y estímulos a la cultura, se crea el Ministerio de la Cultura y se trasladan algunas dependencias. Agosto 7 de 1997. Do 43102 
Los Derechos Culturales. Declaración de Friburgo. Disponible en http://www.culturalrights.net/descargas/ drets_culturals239.pdf

Luis Andrés Fajardo Arturo. Contenido y alcance jurisprudencial del bloque de constitucionalidad en Colombia. Revista Civilizar Ciencias Sociales y Humanas 7(13). 2007. Págs. 15-34.
Rodrigo Uprimny. El BLOQUE DE CONSTITUCIONALIDAD en Colombia. Curso de Formación de PromotoRES/AS DE DERECHOS HUMANOS LIBERTAD SINDICAL Y TRABajo DeCente. Ed: Universidad-ens Colombia. (2005) 